\title{
Characterization of ideals of rhotrices over a ring and its applications
}

\author{
Kailash M. Patil \\ Department of Mathematics, Dharmsinh Desai University \\ Nadiad, Gujarat 387001, India \\ e-mail: kailashpatil2111.mathseddu.ac.in
}

Received: 21 December 2019 Revised: 17 September $2020 \quad$ Accepted: 28 January 2021

\begin{abstract}
We define higher order rhotrices over a commutative unital ring $S$ and obtain a ring $\mathcal{R}_{n}(S)$ of rhotrices of the order $n \in \mathbb{N}$. We characterize the ideals and maximal ideals of $\mathcal{R}_{n}(S)$. As a particular case, we record ideals of rhotrix rings over integers and rhotrix algebras over complex plane $\mathbb{C}$. As an application, we characterize the maximal ideals of the commutative unital Banach algebra $\mathcal{R}_{n}(\mathbb{C})$.
\end{abstract}

Keywords: Rhotrix over a ring, Unital ring, Maximal ideal, Banach algebra.

2010 Mathematics Subject Classification: 15B99, 46J20.

\section{Introduction}

The concept of rhotrices was introduced by Ajibade [1] in 2003 as objects of order higher than $2 \times 2$ and lower than $3 \times 3$ matrices, as an extension of the initiative on matrix-tertions and matrix-noitrets suggested by Atanassov and Shannon [2]. In literature it is realized that with a slight perturbation of the arrangement of a matrix, a rhotrix - a rhomboidal array is obtained. As in [1], a rhotrix of order 3 is defined as

$$
R=\left\langle\begin{array}{ccc}
a & \\
b & h(R) & d \\
e &
\end{array}\right\rangle,
$$

where $a, b, d, e, h(R) \in \mathbb{R} . h(R)$ is called the heart of the rhotrix. For higher order rhotrices, it is difficult to realize a rhotrix as objects of order higher than $(n-1) \times(n-1)$ and lower than $n \times n$ 
matrices. But it will be convenient to see it as a part of $n \times n$ matrix. Thus a rhotrix of order 3 is looked upon as a part of $3 \times 3$ matrix

$$
A_{R}=\left[\begin{array}{ccc}
a & * & d \\
* & h(R) & * \\
b & * & e
\end{array}\right]
$$

rotated through $h(R)$ clockwise by $45^{\circ}$ and removing $*$. The concept was generalized to a rhotrix of order $n$ by Mohammed [7]. He defined a rhotrix $R$ of order $n$ as

$$
R=\mid \begin{array}{ccccccc} 
& & & a_{1} & & & \\
& & a_{2} & a_{3} & a_{4} & & \\
\cdots & \cdots & \cdots & \cdots & \cdots & \\
a_{\frac{t-n+2}{2}} & \cdots & \cdots & a_{\frac{t+1}{2}} & \cdots & \cdots & a_{\frac{t+n}{2}} \\
& \cdots & \cdots & \cdots & \cdots & \cdots &
\end{array}
$$

where $t=\frac{n^{2}+1}{2}$ and $a_{i} \in \mathbb{R}$. Here $h(R)=a_{\frac{t+1}{2}}$ is called the heart of $R$.

We shall denote the rhotrix defined in (1) by $R=\left\langle h(R), a_{i}\right\rangle, 1 \leq i \leq t, i \neq \frac{t+1}{2}$. In what follows, for brevity, when $h(R)$ is mentioned separately, we shall assume tacitly that $1 \leq i \leq t$ and $i \neq \frac{t+1}{2}$.

The set of all rhotrices of order $n$ is denoted by $\mathcal{R}_{n}(\mathbb{R})$. The heart based product on $\mathcal{R}_{n}(\mathbb{R})$ is defined in [7] as shown below. Let $R_{1}=\left\langle h(R), a_{i}\right\rangle, R_{2}=\left\langle h\left(R_{2}\right), b_{i}\right\rangle \in \mathcal{R}_{n}(\mathbb{R}), 1 \leq i \leq t$. Then

$$
R_{1} R_{2}=\left\langle h\left(R_{1}\right) h\left(R_{2}\right), h\left(R_{1}\right) b_{i}+h\left(R_{2}\right) a_{i}\right\rangle .
$$

In passing we note that for a fixed $k \neq \frac{t+1}{2}$ the two dimensional subspace $V=\left\{\left(h(R), a_{k}\right)\right.$ : $\left.R \in \mathcal{R}_{n}(\mathbb{R})\right\}$ becomes a Banach algebra. This and several other multiplications on a two dimensional Banach algebra are investigated in [3].

On the other hand, motivated by matrix multiplication, Sani [10] multiplied two rhotrices of order $n$ in a different way. To realize this we write a rhotrix of order $n$ as a fusion of two matrices, one matrix $\left[a_{i j}\right]$ of the order $\frac{n+1}{2}$ and the other $\left[c_{i j}\right]$ of the order $\frac{n-1}{2}$. Thus a rhotrix of order $n$, written differently, is

$$
\begin{aligned}
& R=\left\langle a_{i j}, c_{l k}\right\rangle
\end{aligned}
$$

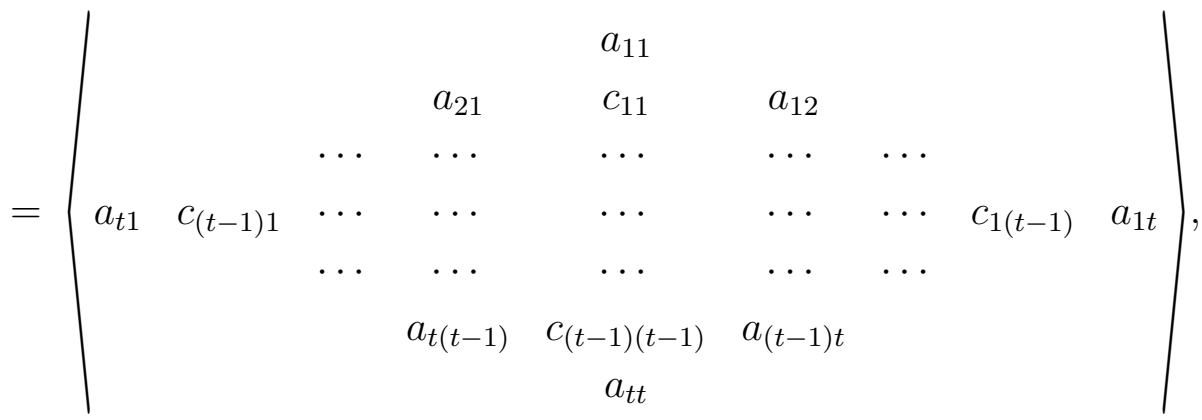

where $t=\frac{n+1}{2}$ and the number of components of $R$ is given by $|R|=\frac{n^{2}+1}{2}$. For two rhotrices $R_{1}=\left\langle a_{i j}, c_{l k}\right\rangle, R_{2}=\left\langle b_{i j}, d_{l k}\right\rangle$, the (noncommutative) product is defined as 


$$
R_{1} \circ R_{2}=\left\langle\sum_{q=1}^{t} a_{i q} b_{q j}, \sum_{q=1}^{t-1} c_{l q} d_{q k}\right\rangle
$$

Mohammed and Okon [8] investigated the noncommutative group structure of general rhotrices of order $n$ with noncommutative product. We consider the commutative product and investigate ring theoretic and algebra theoretic structures of rhotrices by allowing the elements of rhotrices to come from a ring, the set of integers or complex plane. In yet another direction, Isere [4] and [5] defined heartless rhotrices incorporating the even ordered rhotrices. But in the present note, our arrangement is possible only when $n$ is odd. Thus throughout this note, when we refer to $n$ as an order of a rhotrix, $n$ will be assumed to be a positive odd integer.

As mentioned in the above definition of the rhotrix $R$, it is a fusion of two matrices $\left\langle a_{i j}, c_{l k}\right\rangle$, which form a part of an $n \times n$ matrix

$$
A_{R}=\left[\begin{array}{ccccccc}
a_{11} & * & \cdots & \ldots & \cdots & * & a_{1 t} \\
* & c_{11} & \cdots & * & \cdots & c_{1(t-1)} & * \\
\cdots & \cdots & \cdots & \cdots & \cdots & \cdots & \cdots \\
\cdots & * & \cdots & a_{\frac{t+1}{2} \frac{t+1}{2}} & \cdots & * & \cdots \\
\cdots & \cdots & \cdots & \cdots & \cdots & \cdots & \cdots \\
* & c_{(t-1)(1)} & \cdots & * & \cdots & c_{(t-1)(t-1)} & * \\
a_{t 1} & * & \cdots & \cdots & \cdots & * & a_{t t}
\end{array}\right] .
$$

In this case, $\left[a_{i j}\right],\left[c_{l k}\right]$ are called the coupled matrices Sani [11]. Various concepts related to rhotrices are investigated by different researchers. To mention a few, Patil, Singh and Sutaria [9] investigated eigenvalues and eigenvectors of a rhotrix corresponding to a matrix, Sharma and Kanwar [12] have proved Cayley-Hamilton theorem for rhotrices. Usaini and Aminu [13] have defined and investigated the exponential functions on rhotrices.

Ajibade [1] proved that under the heart based multiplication, rhotrices form a semigroup. $\mathrm{He}$ also obtained the necessary and sufficient condition for a rhotrix to be invertible. Mohammed [6] investigates the ring theoretic structure of the same set obtaining ring theoretic results. Both of them restricted themselves to $\mathcal{R}_{3}(\mathbb{R})$, the rhotrices of order 3 . In this paper, we consider rhotrices of order $n$ with entries from a commutative unital ring $S$. Throughout this paper $S$ will denote a commutative unital ring. We denote the identity of $S$ by 1 .

Definition 1.1. By a rhotrix of order $n$ over $S$, we mean a rhotrix

$$
R=\mid \begin{array}{ccccccc} 
& & & a_{1} & & & \\
& & a_{2} & a_{3} & a_{4} & & \\
& \cdots & \cdots & \cdots & \cdots & \cdots & \\
a_{\frac{t-n+2}{2}} & \cdots & \cdots & a_{\frac{t+1}{2}} & \cdots & \cdots & a_{\frac{t+n}{2}} \\
& \cdots & \cdots & \cdots & \cdots & \cdots &
\end{array}
$$

where $t=\frac{n^{2}+1}{2}$ and $a_{i} \in S$. Here $h(R)=a_{\frac{t+1}{2}}$ is the heart of $R$. 
Throughout this note $\mathcal{R}_{n}(S)$ will denote the set of all rhotrices of order $n$ over $S$. Let $R_{1}=\left\langle h\left(R_{1}\right), a_{i}\right\rangle$ and $R_{2}=\left\langle h\left(R_{2}\right), b_{i}\right\rangle$ be in $\mathcal{R}_{n}(S)$. We define

$$
\begin{aligned}
R_{1}+R_{2} & =\left\langle h\left(R_{1}\right)+h\left(R_{2}\right), a_{i}+b_{i}\right\rangle \\
R_{1} R_{2} & =\left\langle h\left(R_{1}\right) h\left(R_{2}\right), h\left(R_{1}\right) b_{i}+h\left(R_{2}\right) a_{i}\right\rangle .
\end{aligned}
$$

In the main result Theorem 2.2, in Section 2, we characterize ideals of the ring $\mathcal{R}_{n}(\mathbb{S})$ of rhotrices over a commutative unital ring $S$. Some very interesting particular cases of rhotrix rings and rhotrix algebras are recorded. As an application of Theorem 2.2, in Section 3, we define Rhotrix Banach algebra and find its maximal ideals and multiplicative linear functionals.

\section{Ideals of $\mathcal{R}_{n}(S)$}

The following is a straight verification using (2).

Proposition 2.1. $\mathcal{R}_{n}(S)$ is a commutative unital ring.

Theorem 2.2. $\mathcal{I} \subset \mathcal{R}_{n}(S)$ is an ideal of $\mathcal{R}_{n}(S)$ if and only if there is a family $\left\{\mathcal{I}_{i}: 1 \leq i \leq t\right\}$ of ideals of $S$ such that $\mathcal{J}_{h}=\mathcal{I}_{\frac{t+1}{2}} \subset \mathcal{I}_{i}$ for every $i$ and

$$
\mathcal{I}=\left\{\left\langle h(R), a_{i}\right\rangle: a_{i} \in \mathcal{I}_{i}, h(R) \in \mathcal{J}_{h}\right\}
$$

Proof. Suppose there is a family $\left\{\mathcal{I}_{i}: 1 \leq i \leq t\right\}$ of ideals of $S$ such that $\mathcal{J}_{h} \subset \mathcal{I}_{i}$ for every $i$ and $\mathcal{I}$ is given by (3). We show that $\mathcal{I}$ is an ideal of $\mathcal{R}_{n}(S)$. Since $\mathcal{I}_{i}$ are ideals of $S$ and since the addition on $\mathcal{R}_{n}(S)$ is pointwise, it is clear that $\mathcal{I}$ is an additive subgroup of $\mathcal{R}_{n}(S)$. Let $R_{1}=\left\langle h\left(R_{1}\right), a_{i}\right\rangle \in \mathcal{R}_{n}(S), R_{2}=\left\langle h\left(R_{2}\right), b_{i}\right\rangle \in \mathcal{I}$, Then for every $i, h\left(R_{1}\right) b_{i} \in S \mathcal{I}_{i} \subset \mathcal{I}_{i}$ because $\mathcal{I}_{i}$ is an ideal of $S$. Also, using the fact that $\mathcal{J}_{h} \subset \mathcal{I}_{i}$, we have $h\left(R_{2}\right) a_{i} \in \mathcal{J}_{h} S \subset \mathcal{I}_{i} S \subset \mathcal{I}_{i}$. Thus $h\left(R_{1}\right) b_{i}+h\left(R_{2}\right) a_{i} \in \mathcal{I}_{i}$. Also, $h\left(R_{1}\right) h\left(R_{2}\right) \in S \mathcal{J}_{h} \subset \mathcal{J}_{h}$ because $\mathcal{J}_{h}$ is an ideal of $S$. Thus $R_{1} R_{2} \in \mathcal{I}$.

Conversely, suppose $\mathcal{I}$ is an ideal of $\mathcal{R}_{n}(S)$. We fix $k$ for the rest of the proof. Define

$$
\mathcal{J}_{h}=\{h(R): R \in \mathcal{I}\}
$$

and

$$
\mathcal{I}_{k}=\left\{a_{k}: R=\left\langle h(R), a_{i}\right\rangle \in \mathcal{I}\right\} .
$$

First we show that $\mathcal{J}_{h}$ is an ideal of $S$. Let $c, d \in \mathcal{J}_{h}$. So, there are $R_{1}=\left\langle h\left(R_{1}\right), a_{i}\right\rangle, R_{2}=$ $\left\langle h\left(R_{2}\right), b_{i}\right\rangle \in \mathcal{I}$ such that $h\left(R_{1}\right)=c$ and $h\left(R_{2}\right)=d$. Then $c+d=h\left(R_{1}\right)+h\left(R_{2}\right)=h\left(R_{1}+R_{2}\right)$. Since $R_{1}+R_{2} \in \mathcal{I}$, it follows that $c+d \in \mathcal{J}_{h}$. Let $c \in \mathcal{J}_{h}$ and $d \in S$. So, there is $R_{1}=$ $\left\langle h\left(R_{1}\right), a_{i}\right\rangle \in \mathcal{I}$ such that $h\left(R_{1}\right)=c$. Define $R_{2}=\left\langle h\left(R_{2}\right), b_{i}\right\rangle \in \mathcal{R}_{n}(S)$ by setting $h\left(R_{2}\right)=d$ and $b_{i}=0$ for every $i$. Then $c d=h\left(R_{1}\right) h\left(R_{2}\right)=h\left(R_{1} R_{2}\right)$. Since $\mathcal{I}$ is an ideal of $\mathcal{R}_{n}(S)$, $R_{1} R_{2} \in \mathcal{I}$. Hence $c d \in \mathcal{J}_{h}$. Thus $\mathcal{J}_{h}$ is an ideal of $S$. Next we show that $\mathcal{I}_{k}$ is an ideal of $S$. Let $c, d \in \mathcal{I}_{k}$. So, there are $R_{1}=\left\langle h\left(R_{1}\right), a_{i}\right\rangle, R_{2}=\left\langle h\left(R_{2}\right), b_{i}\right\rangle \in \mathcal{I}$ such that $a_{k}=c$ and $b_{k}=d$.

Since $\mathcal{I}$ is an ideal, $R_{1}+R_{2} \in \mathcal{I}$. Hence it follows that $c+d=a_{k}+b_{k} \in \mathcal{I}_{k}$. Let $c \in \mathcal{I}_{k}$ and $d \in S$. So, there is $R_{1}=\left\langle h\left(R_{1}\right), a_{i}\right\rangle \in \mathcal{I}$ such that $a_{k}=c$. Define $R_{2}=\left\langle h\left(R_{2}\right), b_{i}\right\rangle \in \mathcal{R}_{n}(S)$ 
by setting $h\left(R_{2}\right)=d$ and $b_{i}=0$ for every $i$. Now the $k$-th coordinate of $R_{1} R_{2}$ is $h\left(R_{1}\right) b_{k}+h\left(R_{2}\right) a_{k}=h\left(R_{1}\right) 0+d c=c d$. Since $\mathcal{I}$ is an ideal of $\mathcal{R}_{n}(S), R_{1} R_{2} \in \mathcal{I}$ giving $c d \in \mathcal{I}_{k}$. Thus $\mathcal{I}_{k}$ is an ideal of $S$. Finally, we show that $\mathcal{J}_{h} \subset \mathcal{I}_{k}$. Let $c \in \mathcal{J}_{h}$. So, there is $R_{1}=\left\langle h\left(R_{1}\right), a_{i}\right\rangle \in \mathcal{I}$ such that $h\left(R_{1}\right)=c$. Define $R_{2}=\left\langle h\left(R_{2}\right), b_{i}\right\rangle \in \mathcal{R}_{n}(S)$ such that $h\left(R_{2}\right)=0$ and $b_{i}=\mathbf{1}$ for every $i$. Now the $k$-th coordinate of $R_{1} R_{2}$ is $h\left(R_{1}\right) b_{k}+h\left(R_{2}\right) a_{k}=c \cdot \mathbf{1}+0 \cdot a_{k}=c$. Since $\mathcal{I}$ is an ideal of $\mathcal{R}_{n}(S), R_{1} R_{2} \in \mathcal{I}$, which gives $c \in \mathcal{I}_{k}$.

We cannot drop the condition that $S$ is unital. The following example shows that it is necessary.

Example 2.3. Consider $\mathcal{R}_{3}(2 \mathbb{Z})$ and

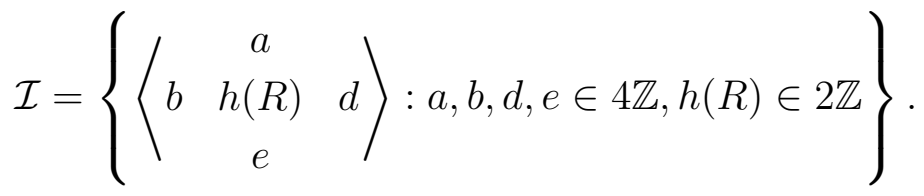

Then one easily verifies that $\mathcal{I}$ is an ideal of $\mathcal{R}_{3}(2 \mathbb{Z})$, however $\mathcal{J}_{h}$ is not a subset of $\mathcal{I}_{1}$.

As an application of the Theorem 2.2, we obtain the characterization of maximal ideals of $\mathcal{R}_{n}(S)$.

Theorem 2.4. An ideal $\mathcal{I}$ of $\mathcal{R}_{n}(S)$ is maximal if and only if $\mathcal{J}_{h}$ is a maximal ideal of $S$ and $\mathcal{I}_{i}=S$ for every $i \neq \frac{t+1}{2}$.

Proof. Suppose $\mathcal{I}_{k} \subsetneq S$. Then we define

$$
\mathcal{K}=\left\{\left\langle h(R), a_{i}\right\rangle: a_{i} \in \mathcal{I}_{i}, \text { for every } i \neq k, h(R) \in \mathcal{J}_{h}, a_{k} \in S\right\} .
$$

Then $\mathcal{I} \subsetneq \mathcal{K}$. Thus it follows that $\mathcal{I}_{k}=S$. Now suppose $\mathcal{J}_{h}$ is not maximal in $S$. So that there is an ideal $\mathcal{K}_{h}$ of $S$ such that $\mathcal{J}_{h} \subsetneq \mathcal{K}_{h}$. Thus allowing the elements of heart to come from $\mathcal{K}_{h}$ instead of $\mathcal{J}_{h}$ as above, we see that maximality of $\mathcal{I}$ is annihilated. Thus the result follows.

In the rest of this section, we give some particular cases of rhotrix rings. In fact, the results are true for rhotrices of order $n$ for any $n \in \mathbb{N}$, but to save the space, we illustrate the same for rhotrices of order 3. For brevity, first we change the notations in this section. The set

$$
\mathcal{I}=\left\{\left\langle\begin{array}{ccc} 
& a_{1} \\
a_{2} & h(R) & a_{4} \\
& a_{5}
\end{array}\right): a_{i} \in m_{i} \mathbb{Z}, \text { for } i=1,2,4,5, h(R) \in m_{3} \mathbb{Z}\right\}
$$

is written as $\left\langle m_{2} \mathbb{Z} \quad \begin{array}{ll}m_{1} \mathbb{Z} \\ m_{3} \mathbb{Z} \\ m_{5} \mathbb{Z}\end{array} m_{4} \mathbb{Z}\right\rangle$.

Also, given $\mathcal{I} \subset R_{3}(\mathbb{Z})$ and $k=1,2,4,5$, we define

$$
\mathcal{I}_{k}=\left\{a_{k}: R=\left\langle h(R), a_{i}\right\rangle \in \mathcal{I}\right\}
$$

and

$$
\mathcal{J}_{h}=\{h(R): R \in \mathcal{I}\} .
$$


Corollary 2.4.1. $\mathcal{I}$ is an ideal of $R_{3}(\mathbb{Z})$ if and only if

$$
\mathcal{I}=\left\langle\begin{array}{lll} 
& m_{1} \mathbb{Z} \\
m_{2} \mathbb{Z} & m_{3} \mathbb{Z} & m_{4} \mathbb{Z} \\
& m_{5} \mathbb{Z}
\end{array}\right.
$$

for some integers $m_{1}, m_{2}, \ldots, m_{5}$ and $m_{k} \mid m_{3}$ for every $k$.

Proof. Though the proof follows from the Theorem 2.2, we give the direct proof. One way proof follows by noting in the following product that $m_{i} \mid\left(a_{3} m_{i} b_{i}+m_{3} b_{3} a_{i}\right)$ because $m_{i} \mid m_{3}$, for every $i=1,2,4,5$ and $a_{1}, \ldots, a_{5}, b_{1}, \ldots, b_{5} \in \mathbb{Z}$, where $A=\left\langle\begin{array}{ccc}a_{1} \\ a_{2} & a_{3} & a_{4} \\ a_{5}\end{array}\right\rangle \in R_{3}(\mathbb{Z})$ and $B=\left\langle m_{2} b_{2} \quad \begin{array}{ll}m_{1} b_{1} \\ m_{3} b_{3} & m_{4} b_{4} \\ m_{5} b_{5}\end{array}\right\rangle \in \mathcal{I}$

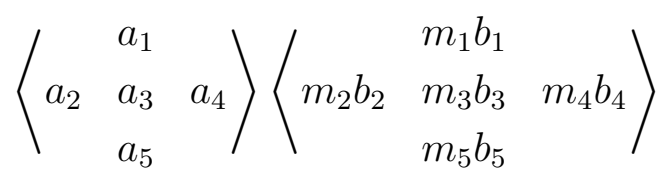

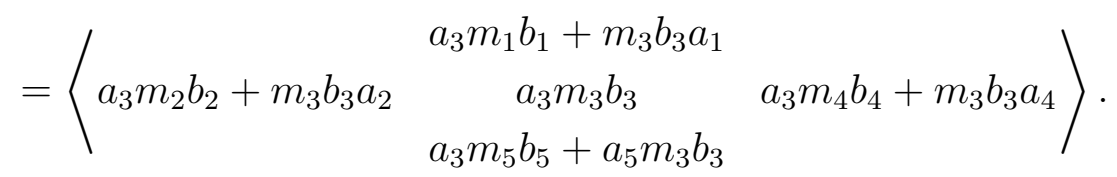

Conversely, suppose $\mathcal{I}$ is an ideal of $R_{3}(\mathbb{Z})$. Following the notations defined before the statement, first we show that $\mathcal{J}_{h}$ as well as $\mathcal{I}_{k}(k=1,2,4,5)$ is an ideal of $\mathbb{Z}$. We fix $k \in\{1,2,4,5\}$. Let $a, b \in \mathcal{I}_{k}$. So, there are $R_{1}=\left\langle h\left(R_{1}\right), a_{i}\right\rangle \in \mathcal{I}, R_{2}=\left\langle h\left(R_{2}\right), b_{i}\right\rangle \in \mathcal{I}$ such that $a=a_{k}$, and $b=b_{k}$. Hence $a-b=a_{k}-b_{k} \in \mathcal{I}_{k}$ because $R_{1}-R_{2}=\left\langle h\left(R_{1}-R_{2}\right), a_{i}-b_{i}\right\rangle \in \mathcal{I}$. Thus $\mathcal{I}_{k}$ is a subgroup of $\mathbb{Z}$. Similarly, $\mathcal{J}_{h}$ is also a subgroup of $\mathbb{Z}$. Hence from the basic result of the algebra and number theory, there are $m_{i} \in \mathbb{Z},(1 \leq i \leq 5)$ such that $\mathcal{I}_{k}=m_{k} \mathbb{Z}$ and $\mathcal{J}_{h}=m_{3} \mathbb{Z}$. Thus we conclude that the ideal $\mathcal{I}$ must be of the form $\mathcal{I}=\left\langle\begin{array}{ll}m_{2} \mathbb{Z} & \begin{array}{ll}m_{1} \mathbb{Z} \\ m_{3} \mathbb{Z} \\ m_{5} \mathbb{Z}\end{array} \quad m_{4} \mathbb{Z}\end{array}\right\rangle$. To settle the final claim, we note that $\left\langle\begin{array}{lll}m_{3} & \\ m_{3} & m_{3} & m_{3} \\ m_{3}\end{array} \quad=\left\langle\begin{array}{ccc}1 & 1 \\ 1 & 1 & 1 \\ 1\end{array}\right\rangle\left\langle\begin{array}{ccc}0 \\ 0 & m_{3} & 0 \\ 0\end{array}\right\rangle \in \mathcal{I}\right.$. This shows that $m_{k} \mid m_{3}$ for every $k$.

\section{Corollary 2.4.2.}

$$
\mathcal{I}=\left\langle\begin{array}{lll} 
& m_{1} \mathbb{Z} & \\
m_{2} \mathbb{Z} & m_{3} \mathbb{Z} & m_{4} \mathbb{Z} \\
& m_{5} \mathbb{Z} &
\end{array}\right\rangle
$$

with $m_{1}, m_{2}, \ldots, m_{5} \in \mathbb{Z}$ is a maximal ideal of $\mathcal{R}_{3}(\mathbb{Z})$ if and only if $m_{1}, m_{2}, m_{4}, m_{5} \in\{-1,1\}$ and $m_{3}$ is prime. 
Proof. One way it is clear that if $m_{1}, m_{2}, m_{4}, m_{5} \in\{-1,1\}$ and $m_{3}$ is prime, then $\mathcal{I}$ is a maximal ideal. Conversely, if $m_{i} \notin\{ \pm 1\}$ for some $i=1,2,4,5$, then $\mathcal{I} \subsetneq\left\langle\begin{array}{cc}\mathbb{Z} & m_{3} \mathbb{Z} \\ \mathbb{Z} & \mathbb{Z}\end{array}\right\rangle R_{3}(\mathbb{Z})$. This contradiction shows that $m_{1}, m_{2}, m_{4}, m_{5} \in\{-1,1\}$. Also if $m_{3}$ is not prime, then choosing a prime factor $p$ of $m_{3}$, we see that $\mathcal{I} \subsetneq\left\langle\begin{array}{ccc}\mathbb{Z} & p \mathbb{Z} & \mathbb{Z} \\ \mathbb{Z} & \subsetneq\end{array}\right) R_{3}(\mathbb{Z})$. This confirms that $m_{3}$ must be prime. This completes the proof.

The following deals with the rhotrix algebra $\mathcal{R}_{n}(\mathbb{F})$, where $\mathbb{F}$ is a field.

\section{Corollary 2.4.3.}

$$
\mathcal{I}=\left\langle\begin{array}{ccc} 
& \mathcal{I}_{1} & \\
\mathcal{I}_{2} & \mathcal{I}_{3} & \mathcal{I}_{4}
\end{array}\right.
$$

is an ideal of $\mathcal{R}_{3}(\mathbb{F})$ if and only if for every $1 \leq i \leq 5 \mathcal{I}_{i}$ is either $\{0\}$ or $\mathbb{F}$. Further, $\mathcal{I}$ is a maximal ideal if and only if $\mathcal{I}_{i}=\mathbb{F}$ for every $i \neq 3$ and $\mathcal{I}_{3}=\{0\}$.

Proof. Arguing in the same way as in the Corollary 2.4.2 and replacing $\mathbb{Z}$ by $\mathbb{F}$, we see that $\mathcal{I}_{k}$ are the ideals of $\mathbb{F}$ for every $k$. Since the ideals of a field $\mathbb{F}$ are $\{0\}$ and $\mathbb{F}$ only, the proof follows.

\section{Rhotrix Banach algebras}

We recall that a Banach algebra is a vector space $\mathcal{A}$ over $\mathbb{R}$ or $\mathbb{C}$ endowed with a complete norm $\|\cdot\|$ and a ring multiplication $(x, y) \in \mathcal{A} \times \mathcal{A} \mapsto x y \in A$ satisfying, for all $x, y, z \in \mathcal{R}_{n}(\mathbb{C})$,

$$
\begin{aligned}
x(y+z) & =x y+x z \\
(y+z) x & =y x+z x \\
\alpha(x y) & =(\alpha x) y=x(\alpha y) \\
\|x y\| & \leq\|x\|\|y\| \\
\|\mathbf{1}\| & =1 .
\end{aligned}
$$

We illustrate the concept of Banach algebras by means putting on record some standard Banach algebras.

Example 3.1. $\quad$ 1. $\mathbb{C}^{n}$ with the coordinatewise operations and the following norm is a commutative unital Banach algebra.

$$
\left\|\left(z_{1}, z_{2}, z_{3} \ldots, z_{n}\right)\right\|_{\infty}=\max \left\{\left|z_{i}\right|: 1 \leq i \leq n\right\},\left(z_{1}, z_{2}, z_{3} \ldots, z_{n}\right) \in \mathbb{C}^{n}
$$

\section{Function Algebras}


(a) For a compact Hausdorff topological space $X$, let $C(X)=\{f: X \rightarrow \mathbb{C}: f$ is continuous on $X\}$. Then with the pointwise operations and the following norm (as known as supnorm) $C(X)$ is a commutative unital Banach algebra.

$$
\|f\|_{\infty}=\sup \{|f(x)|: x \in X\},(f \in C(X)) .
$$

(b) Let $\mathbb{D}=\{z \in \mathbb{C}:|z| \leq 1\}$ and $A(\mathbb{D})=\{f: \mathbb{D} \rightarrow \mathbb{C}: f$ is analytic on the interior of $\mathbb{D}$ and continuous on the boundary of $\mathbb{D}\}$. Then $A(\mathbb{D})$ is a Banach algebra with identity with the supnorm defined as follows.

$$
\|f\|_{\infty}=\sup \{|f(z)|: z \in \mathbb{D}\},(f \in A(\mathbb{D})) .
$$

There are well known Banach algebras of operators on Hilbert spaces, sequence algebras and algebras of differentiable functions on compact manifolds.

The present note gives a new class of Banach algebras, known as rhotrix algebras, that have not yet appeared anywhere in the literature so far.

It is easy to see that $\mathcal{R}_{n}(\mathbb{C})$ is a vector space as well as a ring with heart based multiplication. Note that the identity of $\mathcal{R}_{n}(\mathbb{C})$ is $\mathbf{1}=\left\langle h(\mathbf{1}), a_{i}\right\rangle$, where $h(\mathbf{1})=1$ and $a_{i}=0$ for every $i$.

Definition 3.2. Let $n \in \mathbb{N}$. For $\left\langle h(R), a_{i}\right\rangle \in \mathcal{R}_{n}(\mathbb{C})$, we define the norm

$$
\left\|\left\langle h(R), a_{i}\right\rangle\right\|=|h(R)|+\max _{i}\left|a_{i}\right| .
$$

Lemma 3.3. $\mathcal{R}_{n}(\mathbb{C})$ is a Banach algebra with the norm defined in (4).

Proof. Clearly, $\|\cdot\|$ is a vector space norm on $\mathcal{R}_{n}(\mathbb{C})$, i.e., for $R_{1}, R_{2} \in \mathcal{R}_{n}(\mathbb{C})$, and $\alpha \in \mathbb{C}$,

$$
\begin{aligned}
\left\|R_{1}\right\| & \geq 0 \text { and }\left\|R_{1}\right\|=0 \text { iff } R_{1}=0 \\
\left\|R_{1}+R_{2}\right\| & \leq\left\|R_{1}\right\|+\left\|R_{2}\right\| \\
\left\|\alpha R_{1}\right\| & =|\alpha|\left\|R_{1}\right\| .
\end{aligned}
$$

To prove the submultiplicativity of the norm, let $R_{1}=\left\langle h\left(R_{1}\right), a_{i}\right\rangle, R_{2}=\left\langle h\left(R_{2}\right), b_{i}\right\rangle \in \mathcal{R}_{n}(\mathbb{C})$. Then

$$
\begin{aligned}
\left\|R_{1} R_{2}\right\|= & \left|h\left(R_{1}\right) h\left(R_{2}\right)\right|+\max _{i}\left\{\left|h\left(R_{1}\right) b_{i}+h\left(R_{2}\right) a_{i}\right|\right\} \\
\leq & \left|h\left(R_{1}\right)\right|\left|h\left(R_{2}\right)\right|+\max _{i}\left\{\left|h\left(R_{1}\right) b_{i}\right|+\left|h\left(R_{2}\right) a_{i}\right|\right\} \\
\leq & \left|h\left(R_{1}\right)\right|\left|h\left(R_{2}\right)\right|+\max _{i}\left\{\left|h\left(R_{1}\right) b_{i}\right|\right\}+\max _{i}\left\{\left|h\left(R_{2}\right) a_{i}\right|\right\} \\
= & \left|h\left(R_{1}\right)\right|\left|h\left(R_{2}\right)\right|+\left|h\left(R_{1}\right)\right| \max _{i}\left\{\left|b_{i}\right|\right\}+\left|h\left(R_{2}\right)\right| \max _{i}\left\{\left|a_{i}\right|\right\} \\
\leq & \left|h\left(R_{1}\right)\right|\left|h\left(R_{2}\right)\right|+\left|h\left(R_{1}\right)\right| \max _{i}\left\{\left|b_{i}\right|\right\}+\left|h\left(R_{2}\right)\right| \max _{i}\left\{\left|a_{i}\right|\right\} \\
& +\max _{i}\left\{\left|a_{i}\right|\right\} \max _{i}\left\{\left|b_{i}\right|\right\} \\
= & \left|h\left(R_{1}\right)\right|\left(\left|h\left(R_{2}\right)\right|+\max _{i}\left\{\left|b_{i}\right|\right\}\right)+\max _{i}\left\{\left|a_{i}\right|\right\}\left(\left|h\left(R_{2}\right)\right|+\max _{i}\left\{\left|b_{i}\right|\right\}\right) \\
= & \left(\left|h\left(R_{1}\right)\right|+\max _{i}\left\{\left|a_{i}\right|\right\}\right)\left(\left|h\left(R_{2}\right)\right|+\max _{i}\left\{\left|b_{i}\right|\right\}\right) \\
= & \left\|R_{1}\right\|\left\|R_{2}\right\| .
\end{aligned}
$$

This completes the proof. 
We demonstrate the computations in the following example.

Example 3.4. Let $R_{1}=\left\langle\begin{array}{lll}1 & \\ 1 & 2 & 1 \\ 1 & \end{array}\right\rangle$ and $R_{2}=\left\langle\begin{array}{lll}5 & \\ 1 & 1 & 1 \\ 1\end{array}\right\rangle$. Then

$$
\begin{aligned}
& R_{1} R_{2}=\left\langle\begin{array}{ccc} 
& 2 \times 5+1 \times 1 & \\
2 \times 1+1 \times 1 & 2 \times 1 & 2 \times 1+1 \times 1 \\
& 2 \times 1+1 \times 1
\end{array}\right. \\
& =\left\langle\begin{array}{ccc}
11 & \\
3 & 2 & 3
\end{array}\right\rangle
\end{aligned}
$$

Also,

$$
\begin{aligned}
\left\|R_{1}\right\| & =|2|+\max \{1,1,1,1\}=3 \\
\left\|R_{2}\right\| & =|1|+\max \{5,1,1,1\}=6 \\
\left\|R_{1} R_{2}\right\| & =|2|+\max \{11,3,3,3\}=13 .
\end{aligned}
$$

Hence,

$$
\left\|R_{1} R_{2}\right\| \leq\left\|R_{1}\right\|\left\|R_{2}\right\|
$$

Remark 3.5. The most natural supnorm is not carried over to $\mathcal{R}_{n}(\mathbb{C})$ because of its heart based multiplication. In fact the submultiplicativity of supnorm fails as can be seen from the following.

For rhotrix $R_{1}=\left\langle h\left(R_{1}\right), a_{i}\right\rangle \in \mathcal{R}_{3}(\mathbb{C})$, consider the most natural candidate as a norm on $\mathcal{R}_{n}(\mathbb{C}),\left\|\left\langle\begin{array}{lll} & a_{1} & \\ a_{2} & a_{3} & a_{4}\end{array}\right\rangle\right\|_{\infty}=\max \left|a_{i}\right|$. Let $R_{1}=\left\langle\begin{array}{ccc}1 & \\ 1 & 2 & 1 \\ 1 & a_{5}\end{array}\right\rangle$ and $R_{2}=\left\langle\begin{array}{ccc}5 & \\ 1 & 1 & 1\end{array}\right\rangle$. Then $\left\|R_{1} R_{2}\right\|_{\infty}=11$, whereas $\left\|R_{1}\right\|_{\infty}\left\|R_{2}\right\|_{\infty}=10$. Thus submultiplicativity fails. As already noted in the Corollary $2.4 .3, \mathcal{R}_{n}(\mathbb{C})$ has only one maximal ideal, that is,

$$
\mathcal{I}=\left\{R \in \mathcal{R}_{n}(\mathbb{C}): h(R)=0\right\}
$$

Using the one-to-one correspondence between the maximal ideals of a Banach algebra and its multiplicative linear functionals, we conclude the following.

Theorem 3.6. $\mathcal{R}_{n}(\mathbb{C})$ admits only one multiplicative linear functional, viz. $\varphi: \mathcal{R}_{n}(\mathbb{C}) \rightarrow \mathbb{C}$, defined by $\varphi(R)=h(R),\left(R \in \mathcal{R}_{n}(\mathbb{C})\right)$.

\section{Acknowledgements}

The author is grateful to the Dharmsinh Desai University for all research facilities. The author gratefully acknowledges the suggestions made by the referees improving the readability of the paper. 


\section{References}

[1] Ajibade, A. O. (2003). The concept of rhotrix in mathematical enrichment. International Journal of Mathematical Education in Science and Technology, 34(2), 175-179.

[2] Atanassov, K. T., \& Shannon, A. G. (1998). Matrix-Tertions and Matrix-Noitrets: Exercises in Mathematical Enrichment. International Journal of Mathematical Education in Science and Technology, 29, 898-903.

[3] Bhatt, S. J., \& Karia, D. J. (1998). A note on Banach algebras without order. Prajna Journal of Sardar Patel University, 8(1), 30-32.

[4] Isere, A. O. (2018). Even dimensional rhotrix. Notes on Number Theory and Discrete Mathematics, 24(2), 125-133.

[5] Isere, A. O. (2019). Representation of higher even-dimensional rhotrix. Notes on Number Theory and Discrete Mathematics, 25(1), 206-219.

[6] Mohammed, A. (2009). A remark on the classifications of rhotrices as abstract structures. International Journal of Physical Sciences, 4(9), 496-499.

[7] Mohammed, A. (2011). Theoretical Development and Applications of Rhotrices. PhD thesis, Ahmadu Bello University, Zaria.

[8] Mohammed, A., \& Okon, U. E. (2016). On subgroups of non-commutative general rhotrix group. Notes on Number Theory and Discrete Mathematics, 22(2), 72-90.

[9] Patil, K. M., Singh, H. P., \& Sutaria, K. A. (2015). The eigen values of any given $3 \times 3$ matrix via eigen values of its corresponding rhotrix. International Journal of Computer and Mathematical Sciences, 4(11), 1-4.

[10] Sani, B. (2007). The row-column multiplication of high dimensional rhotrices. International Journal of Mathematical Education in Science and Technology, 38(5), 657-662.

[11] Sani, B. (2008). Conversion of a rhotrix to a 'coupled matrix'. International Journal of Mathematical Education in Science and Technology, 39(2), 244-249.

[12] Sharma, P. L. \& Kanwar, R. K. (2012). The Cayley-Hamilton theorem for rhotrices. International Journal Mathematics and Analysis, 4(1), 171-178.

[13] Usaini, S. \& Aminu, A. (2017). Exponential function of rhotrices. International Journal of Mathematics and Statistics, 18(1), 21-29. 